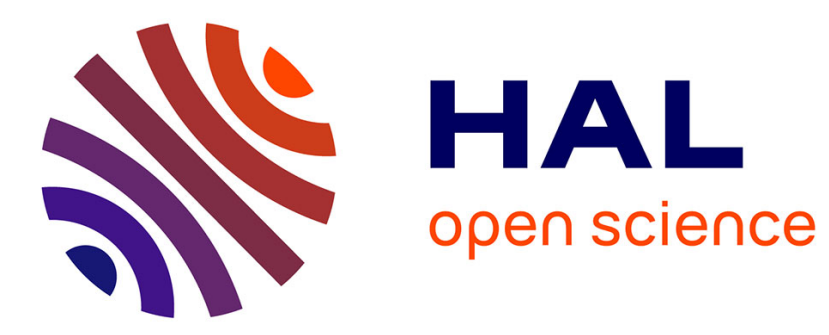

\title{
Craquelures dans les couches picturales des peintures d'art
}

\author{
Ludovic Pauchard, Veronique Lazarus, Bérengère Abou, Ken Sekimoto, \\ Geneviève Aitken, Christian Lahanier
}

\section{To cite this version:}

Ludovic Pauchard, Veronique Lazarus, Bérengère Abou, Ken Sekimoto, Geneviève Aitken, et al.. Craquelures dans les couches picturales des peintures d'art. 2007, pp.5-9. hal-00158845

\section{HAL Id: hal-00158845 \\ https://hal.science/hal-00158845}

Submitted on 29 Jun 2007

HAL is a multi-disciplinary open access archive for the deposit and dissemination of scientific research documents, whether they are published or not. The documents may come from teaching and research institutions in France or abroad, or from public or private research centers.
L'archive ouverte pluridisciplinaire HAL, est destinée au dépôt et à la diffusion de documents scientifiques de niveau recherche, publiés ou non, émanant des établissements d'enseignement et de recherche français ou étrangers, des laboratoires publics ou privés. 


\section{Craquelures dans les couches picturales des peintures d'art}

Ludovic Pauchard ${ }^{1}$ (pauchard@fast.u-psud.fr), Véronique Lazarus ${ }^{2}$,

Bérengère $\mathrm{Abou}^{3}$, Ken Sekimoto ${ }^{3}$, Geneviève Aitken ${ }^{4}$, Christian Lahanier ${ }^{4}$

1 - Laboratoire Fluides, Automatique et Systèmes Thermiques, Université Paris 11, 91405 Orsay

2 - Laboratoire de Modélisation en Mécanique, Université Pierre et Marie Curie, 75005 Paris

3 - Laboratoire Matière et Systèmes Complexes, Université Denis Diderot, 75005 Paris

4 - Centre de Recherche et de Restauration des Musées de France, Palais du Louvre, 75001 Paris

Les craquelures dans

les couches picturales

sont depuis longtemps

un moyen de juger

l'authenticité des

peintures d'art et de

retracer la manière

dont l'artiste a œuvré.

Nous avons étudié les

figures de craquelures

dans la série de peintures de Georges de La Tour

consacrée aux Apôtres,

pour laquelle peintures

de l'artiste et copies

n'ont pas été clairement

dissociées jusqu'à présent.

Nous nous sommes

basés sur des expériences modèles de séchage de

suspensions colloïdales,

qui aident à comprendre

les morphologies des

craquelures observées

sur les œuvres réelles.

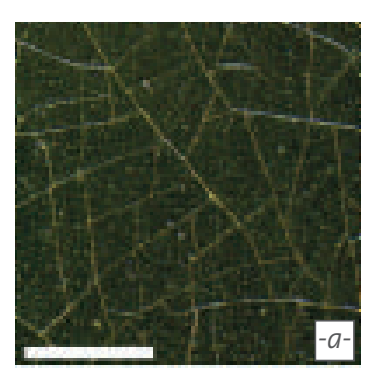

Les études scientifiques appliquées aux peintures d'art ont pour principaux objectifs de donner des éléments de datation et d'authentification et d'analyser les techniques employées par les artistes. Pour cela, les méthodes d'examen des peintures sont principalement basées, d'une part, sur des analyses par prélèvement permettant, entre autres, une identification des pigments employés et, d'autre part, sur des études par traitement d'images. Si ces deux catégories d'examen sont complémentaires, la première reste locale et destructive, tandis que la seconde donne des renseignements sur l'ensemble de l'œuvre et préserve son intégrité. Les études par traitement d'image révèlent avec précision les morphologies des craquelures qui se sont développées dans la peinture. On sait depuis longtemps que ces figures de craquelures, bien qu'indésirables, présentent un intérêt crucial pour juger de l'authenticité d'une peinture. Mais ce n'est que très récemment qu'on cherche à les relier au matériau qui constitue la couche picturale, ainsi qu'aux méthodes employées par l'artiste [1].

Les caractéristiques des morphologies de craquelures sont en effet liées à la nature de la matière picturale (pigments, vernis, enduits de préparation) et au mode de conservation, représentatifs de l'époque et de l'artiste.

Une classification des figures de craquelures montre leur grande variété [2]. Néanmoins, malgré leur complexité apparente, leur formation obéit à certaines règles. Elles sont en effet les empreintes laissées par les contraintes ${ }^{\star}$ mécaniques, accumulées au cours du temps dans la couche picturale et qui se dissipent lors de la formation des craquelures. De ce fait, elles nous renseignent sur ces contraintes, ainsi que sur leurs origines : fragilisation au cours du séchage, sollicitations mécaniques (déplacements ou forces), vieillissement.

Nous nous sommes intéressés récemment à une série incomplète de dix Apôtres et un Christ bénissant, œuvre de Georges de La Tour (1593-1652), conservée au musée Toulouse-Lautrec d'Albi [1]. Les toiles originales figuraient dès 1698 dans la cathédrale Sainte Cécile d'Albi. Mais la plupart des originaux ont laissé la place à des copies à une date qui reste inconnue. Ainsi, parmi les toiles conservées, seules Saint Jacques le Mineur et Saint Jude Thaddée (représentée en image de couverture) seraient des œuvres autographes. De ce fait, les questions suivantes se posent : s'agit-il de copies faites par un autre artiste ? De variantes réalisées par Georges de La Tour ou par son atelier ? Comment distinguer les originaux des copies, des variantes? Pour y répondre, nous avons classé cette série de toiles suivant les différentes morphologies de craquelures observées. Quelques exemples sont donnés sur la figure 1, où l'on peut observer des réseaux plus ou moins denses de craquelures lisses ou sinueuses, ou des craquelures en germes isolés.

Figure 1 : Quelques morphologies de craquelures tirées de la série des Apôtres.

(a) Réseau dense de craquelures lisses formant des polygones plus ou moins réguliers, caractéristiques d'une couche rigide dans une région sombre de Saint Jacques le Mineur.

(b) Craquelures sinueuses dans une région sombre de Saint André.

(c) Réseau peu dense de longues craquelures sinueuses dans les carnations de Saint Thomas.

(d) Craquelures en germes isolés dans les carnations de Saint Matthias.

(Chaque cliché a la même dimension ; longueur de la barre du cliché (a) : 1 cm). 
Figure 2 : Exemples de craquelures d'origine mécanique. (a) Craquelures formant des portions de spirale, sur la toile représentant Saint Matthias. (b) Intersection de deux réseaux de longues craquelures parallèles dans la peinture de Saint Jacques le Majeur. (c) Craquelures longues, fines et parallèles entre elles, sur la toile représentant Saint Jacques le Mineur (certaines craquelures ont été surimprimées pour guider l'œil). (c) C2RMF (P. Cotte, R. Pillay).
D'autres craquelures sont organisées de manière ordonnée, comme le montre la figure 2.

L'étude des morphologies de craquelures sur système modèle peut aider à comprendre celles que l'on observe sur les œuvres réelles. Dans ce qui suit, nous mettons en évidence les contraintes mécaniques induites lors du séchage d'un système modèle, l'influence de la composition des couches d'une part et de leur épaisseur d'autre part sur les morphologies de craquelures. Quelques éléments de réponses seront ensuite donnés quant au regroupement de certaines peintures de la série des Apôtres à partir des figures de craquelures.

\section{Origines des craquelures dans les couches picturales}

La présence de craquelures traduit l'existence de tensions mécaniques importantes qui se sont dissipées dans la couche picturale. Ces craquelures peuvent avoir de multiples origines. Nous en distinguerons deux types, caractérisés par deux échelles de temps très différentes : d'une part les craquelures dues à la consolidation de la couche picturale, apparaissant lors du séchage ; d'autre part, les craquelures " d'âge » qui se sont produites ultérieurement, résultat d'un vieillissement de la couche elle-même ou de tensions appliquées à la toile.

Le premier type de craquelures peut être influencé par le procédé d'application de la peinture : la contrainte imposée sur la couche par le pinceau induit une direction privilégiée aux craquelures qui apparaissent par la suite [3] ou bien une fragilisation locale qui pourra provoquer des craquelures. Après application de la peinture (particules de pigments, de dimensions inférieures au micron, en suspension dans un diluant) sur le support, la couche sèche sous l'effet de l'évaporation des diluants. Les pigments constituant la couche se concentrent alors progressivement, formant une matrice solide poreuse dont les interstices sont remplis du diluant (fig. 3). Au cours de l'évaporation du diluant, une rétraction progressive de la matrice solide poreuse a lieu. Mais cette rétraction n'est pas libre : elle est limitée par l'adhésion de la matrice poreuse au support ou à une sous-couche. Il s'ensuit des tensions dans la couche qui s'opposent à la rétraction. Lorsque ces contraintes mécaniques, qui augmentent au cours du séchage, atteignent une valeur critique, on observe la nucléation de craquelures à partir des défauts de la couche picturale (hétérogénéités en surface ou volume, aspérités...). Ceci permet de relâcher les contraintes mécaniques.

Le second type de craquelures, que nous qualifions de craquelures "d'âge ", permettent d'obtenir certaines informations sur l'histoire de la peinture. Elles peuvent résulter d'un vieillissement chimique de la couche picturale elle-même. Par opposition au processus de consolidation par séchage, souvent qualifié de vieillissement physique, le vieillissement chimique est la conséquence de différents processus impliquant une modification de la structure chimique des constituants de la couche picturale et de leurs interactions. Citons, par exemple, l'effet de l'oxygène de l'air, l'action de la chaleur, les variations du taux d'humidité relative ou bien l'altération due à la lumière (rayonnement UV). Ce vieillissement chimique conduit à des réarrangements lents
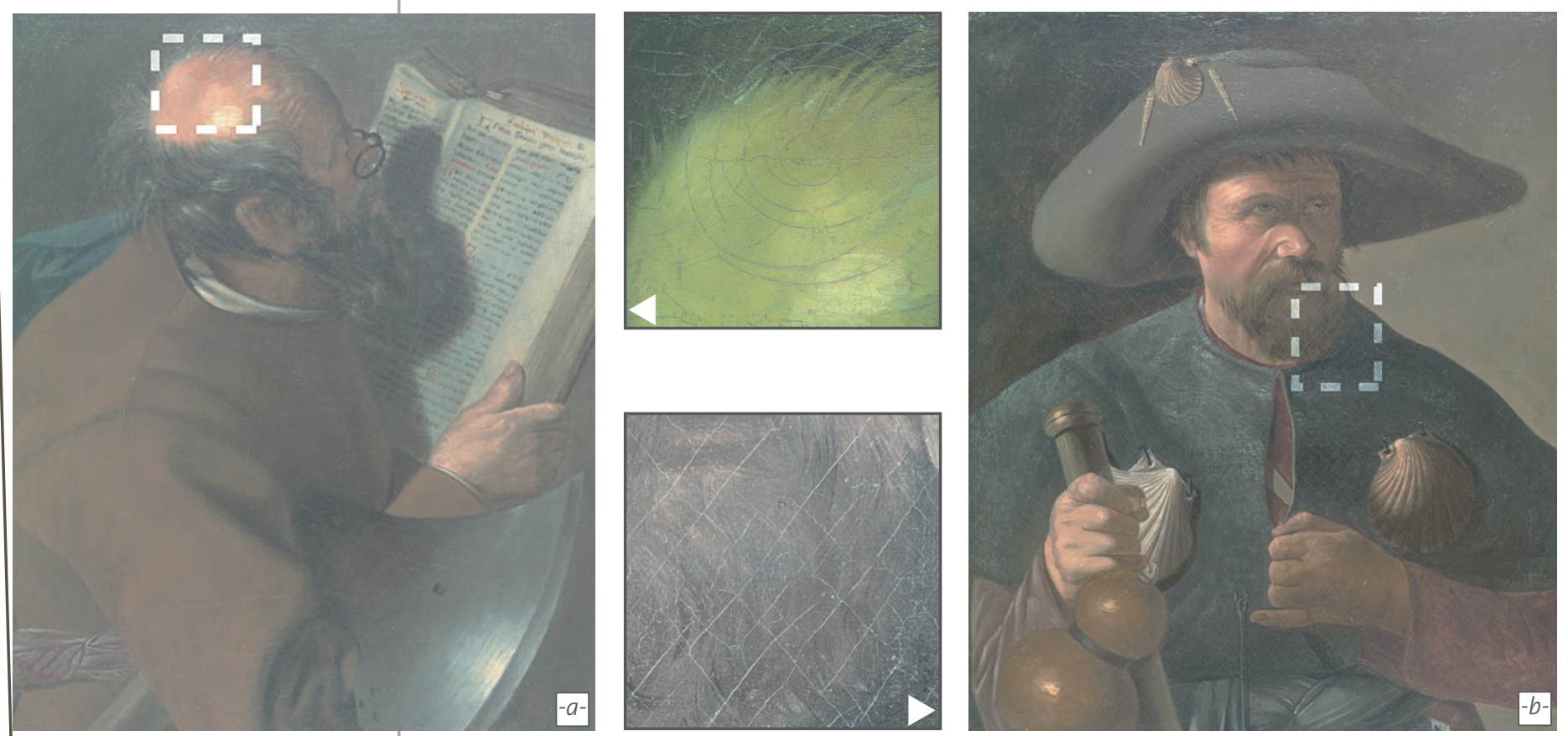
de la microstructure des couches picturales, qui modifient leur comportement mécanique macroscopique. Des craquelures « d'âge » peuvent alors nucléer dans la couche lorsque les contraintes mécaniques sont trop importantes. Elles sont généralement plus fines que les craquelures liées au séchage.

Dans cette classe de craquelures, nous rangeons également celles d'origine purement mécanique, dues par exemple à des chocs ou à des tensions appliquées à la toile en vue de la restaurer. Différentes causes de ce type peuvent être distinguées dans la série des Apôtres :

- de nombreuses toiles présentent des régions où existent des craquelures circulaires ou formant des portions de spirale. Un exemple est donné sur la peinture de Saint Matthias (fig. 2a). Ces craquelures sont probablement dues à des chocs survenus perpendiculairement au plan de la couche ;

- la peinture de Saint Jacques le Majeur présente clairement l'intersection de deux réseaux de longues craquelures parallèles entre elles et équidistantes sur la globalité de la couche picturale (fig. 2b). La direction moyenne de chaque réseau est proche des diagonales du tableau. Ceci suggère que des tensions mécaniques ont été appliquées à la toile, sans doute dans le but d'une restauration (procédé de rentoilage ${ }^{\star}$ ou de transposition de toile sur toile ${ }^{\star}$ ) ;

- les longues craquelures, fines, parallèles entre elles et horizontales, présentes dans la couche picturale de Saint Jacques le Mineur (fig. 2c) suggèrent, quant à elles, que la toile a subi un enroulement.

Finalement, cette classe de craquelures "d'âge " inclue également celles dues à des changements climatiques. Les variations de

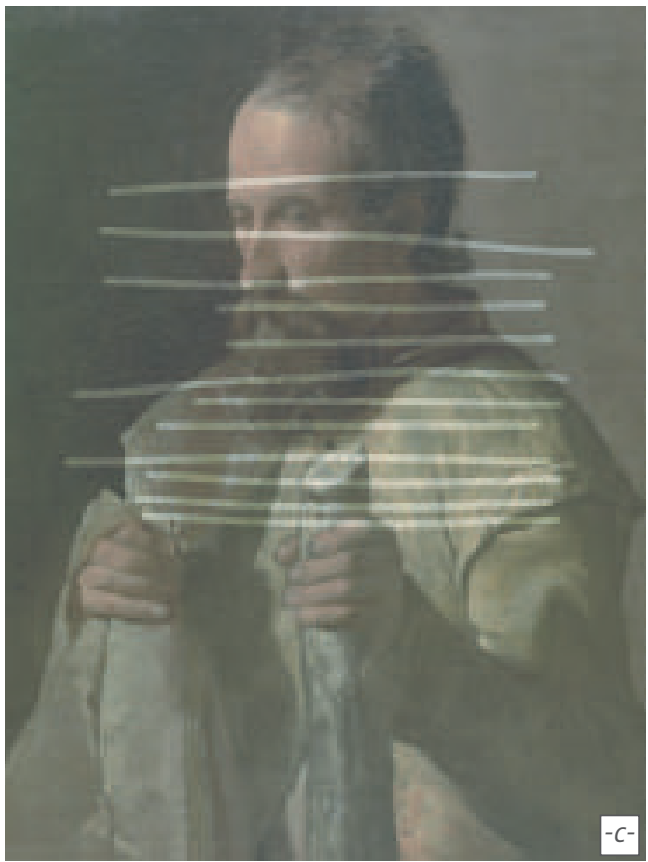

l'hygrométrie et de la température, auxquelles sont exposés les tableaux, peuvent être une source de contrainte mécanique, rendant alors instable le réseau de craquelures. La couche picturale, microporeuse, absorbe l'humidité du milieu environnant, qui peut s'évaporer ensuite si l'hygrométrie diminue. L'évaporation induira alors des tensions dans la couche picturale, conduisant à la formation de nouvelles craquelures.

\section{Modélisation expérimentale des craquelures de séchage}

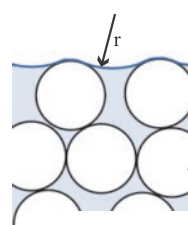

$-a-$

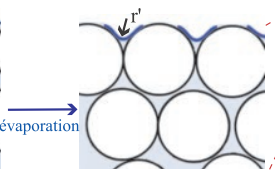

$-b-$

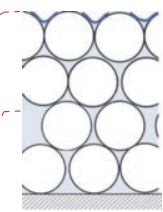

$-c-$
Afin de modéliser la formation de la couche picturale, nous considérons des suspensions de billes colloïdales (de taille inférieure au micron) dont les propriétés mécaniques different en fonction de leur constitution physico-chimique. Au cours de l'évaporation du solvant, comme décrit plus haut, les billes se rapprochent et forment une matrice poreuse solide qui se rétracte progressivement (fig. 3). Cette rétraction est contrariée par l'adhésion au support, ce qui se traduit par l'apparition de contraintes internes et (éventuellement) de craquelures.

Les contraintes mécaniques de rétraction se développant au cours du séchage peuvent être mises en évidence à l'aide du dispositif suivant. Une couche de suspension colloïdale est déposée sur une lame métallique flexible, dont les propriétés mécaniques sont parfaitement connues : l'une de ses extrémités est fixe, tandis que l'autre est libre. Au cours de la consolidation et de la rétraction de la couche, le système lame/couche fléchit (fig. 4-I) ; la mesure de la déflexion de la lame, qui reste dans le domaine élastique, nous permet d'obtenir l'évolution des contraintes moyennes dans la couche [4].

Les craquelures se présentent sous des formes différentes selon la vitesse de séchage de la couche, l'adhésion de celle-ci sur le support, la composition de la couche et son épaisseur. On constate en effet qu'une couche consolidée lentement présente moins de craquelures qu'une couche consolidée rapidement, et que plus l'adhésion est importante, plus il y a de craquelures. Quant à l'influence de la composition et de l'épaisseur, elle est étudiée plus en détail dans ce qui suit.
Figure 3 : Représentation schématique de la formation d'une matrice poreuse au cours du séchage d'une suspension de billes colloïdales. Sous l'effet de la pression capillaire*, les billes se rapprochent en surface (a), formant ainsi une matrice solide dont les interstices sont remplis de solvant (b). Les interfaces solvant/air localisées à la surface de la couche (ligne bleue du schéma (a)) se courbent alors de plus en plus (b), conduisant à une dépression dans le liquide occupant les interstices. Ceci entraîne une rétraction progressive et importante de la matrice poreuse sous l'effet des pressions capillaires. L'adhésion sur un support (c) limite cette rétraction, induisant des contraintes de tension dans la matrice. Ces dernières peuvent atteindre des valeurs très importantes, auxquelles la couche ne peut répondre que par la formation de craquelures.

\section{Nomenclature}

\section{- Contrainte}

notion de la mécanique des milieux continus, permettant de décrire les efforts surfaciques internes à un matériau. Les contraintes sont associées à des déformations élastiques. Lorsqu'elles excèdent une valeur critique, il en résulte des endommagements mécaniques, tels les craquelures.

- Pression capillaire :

variation de pression induite par la courbure d'une interface. Elle est donnée par la relation de Laplace, $P_{c a p}=-2 \gamma_{L V} / r$, où $\gamma_{L V}$ désigne la tension superficielle solvant/air et $r$ le rayon de courbure des interfaces solvant/air. La valeur de $r$ est régie par la taille des pores, donc par celle des billes colloïdales : plus cette dernière sera faible, plus la pression capillaire sera grande. Pour des billes de $20 \mathrm{~nm}$ de diamètre, la pression capillaire peut atteindre $10^{7} \mathrm{~Pa}$.

\section{- Rentoilage :}

technique pratiquée en France depuis le XVII ${ }^{\mathrm{e}}$ siècle. Pour renforcer la toile originale, fragilisée par le vieillissement des fibres et par divers accidents, une toile neuve est apposée au revers à l'aide d'un adhésif à base de colle de farine ou de cire-résine.

- Transposition de toile sur toile : opération drastique qui consiste à éliminer par le revers une toile extrêmement dégradée et à la remplacer par une toile neuve. 
Figure 4 : Expériences modèles mettant en évidence les contraintes moyennes de rétraction au cours de la consolidation de couches de différentes compositions.

(I) Schéma du montage en vue de profil. (II) Évolution des contraintes moyennes pour des couches de billes de latex, d'épaisseur initiale $1 \mathrm{~mm}$, présentant des propriétés mécaniques différentes : couche composée de billes dures (a), d'un mélange de $80 \%$ de billes dures et de $20 \%$ de billes $\mathrm{mol}$ les (b), et uniquement de billes molles (c). Une coupe schématique d'un empilement de billes modèles a été représentée pour les deux cas extrêmes (a) et (c).

(III) La morphologie typique de craquelures correspondant au système constitué de billes dures est présentée sur l'image (a), tandis que celle correspondant au mélange de billes dures et molles est présentée sur l'image (b). Aucune craquelure n'apparaît dans le cas d'une couche constituée uniquement de billes molles.
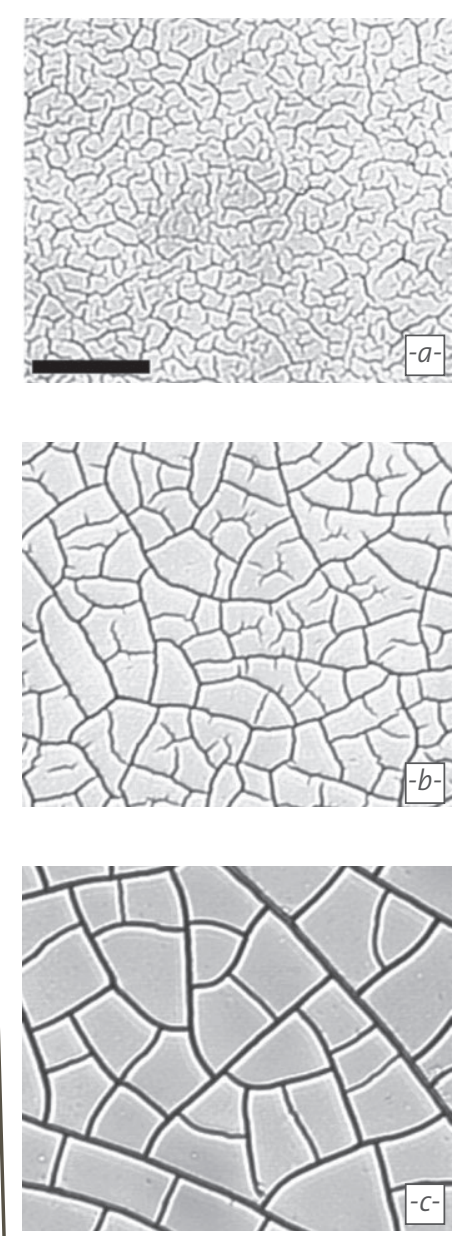

Figure 5 : Expériences modèles présentant les morphologies de craquelures dans des couches de billes de silice de différentes épaisseurs. (a) Pour des couches très fines germes de craquelures isolés. (b) Pour des couches d'épaisseur intermédiaire : de longues craquelures se forment puis coexistent avec des germes sinueux. (c) Pour des couches épaisses : réseau de craquelures lisses formées de manière hiérarchique.

(Chaque image a la même dimension longueur de la barre de l'image (a) : 20 нm).

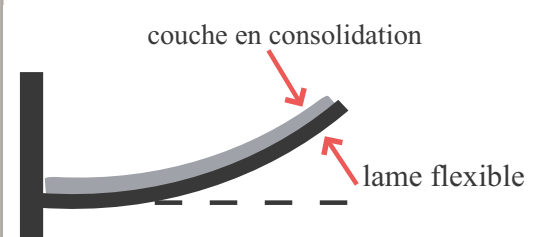

schéma du montage
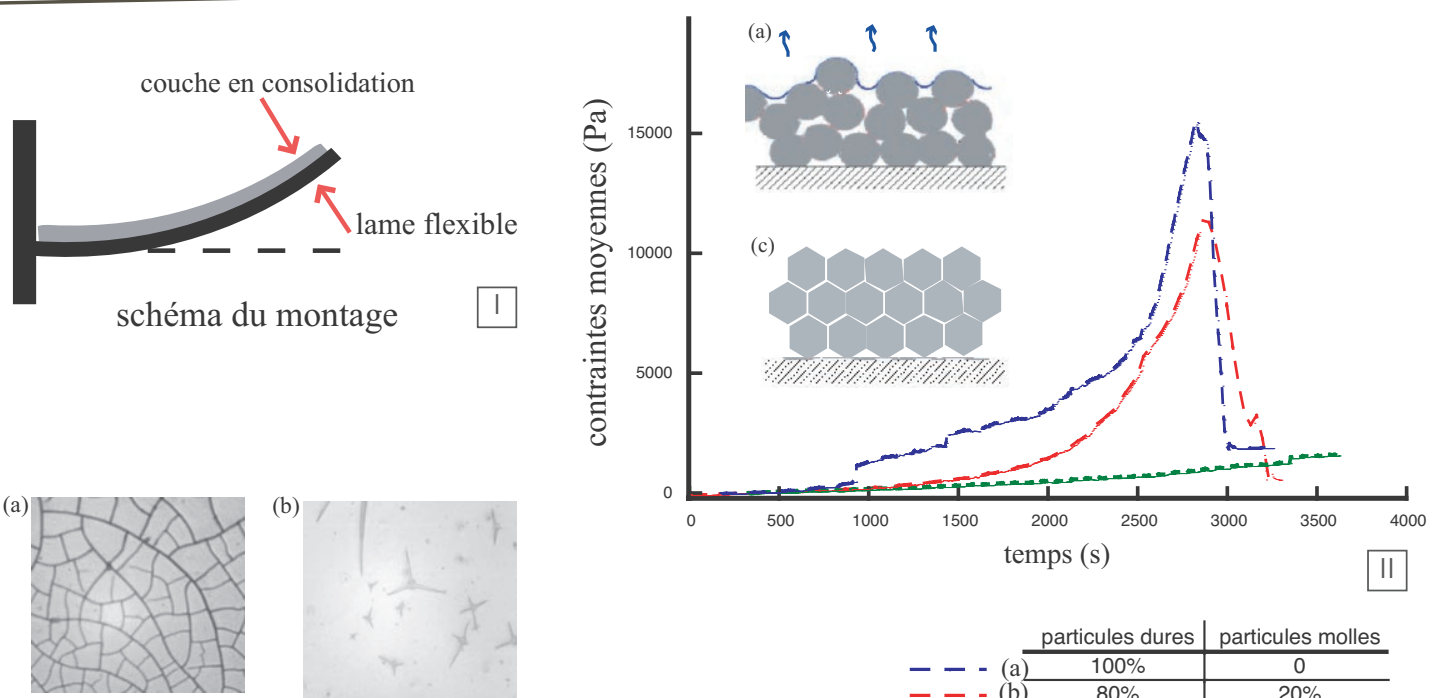

III

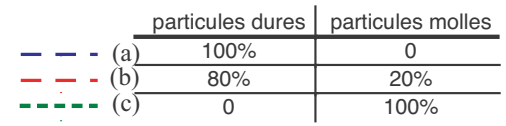

\section{Influence de la composition}

Nous avons entrepris l'étude de couches constituées de deux types de billes colloïdales (billes de latex de différentes températures de transition vitreuse) dans des pourcentages contrôlés : des billes " molles ", déformables, et des billes « dures ", indéformables [5].

- Prenons le cas d'une suspension colloïdale constituée uniquement de billes " dures ». Au cours du séchage de la couche, les contraintes mécaniques augmentent jusqu'à une valeur maximale (courbe bleue de la figure 4-II). Des craquelures apparaissent alors (fig. 4-IIIa), permettant la relaxation de ces contraintes.

- En revanche, pour une couche formée à partir d'une suspension ne comportant que des billes molles, les contraintes mécaniques générées au cours du séchage par la pression capillaire sont essentiellement dissipées par la déformation des billes. Les contraintes mécaniques résiduelles n'augmentent que légèrement (courbe verte de la figure 4-II). Leur valeur est suffisamment faible pour ne pas conduire à la formation de craquelures : la couche reste homogène.

- Utilisons maintenant une couche formée par un mélange de billes dures et de billes molles. Ces dernières se déforment sous l'effet de la rétraction induite par la pression capillaire, relaxant ainsi partiellement les contraintes internes. Par conséquent, ces contraintes augmentent jusqu'à une valeur maximale moins importante que dans le cas d'une couche constituée uniquement de billes dures, tout en restant supérieure à celle correspondant à des billes molles (voir courbe rouge du graphe de la figure 4-II).

On observe expérimentalement que les craquelures se forment pour une concentration seuil de billes molles inférieure à 30\% en masse. Au voisinage de cette concentration seuil, les craquelures forment des germes isolés (figure 4-III b).
Au-dessus, le nombre de germes de craquelures diminue avec la concentration de billes molles présentes, jusqu'à devenir inexistant.

\section{Influence de l'épaisseur de la couche}

Afin de quantifier cet effet, le système modèle que nous considérons maintenant est uniquement constitué de billes "dures ». Les expériences modèles sont basées sur le séchage de ces suspensions collö̈dales dans des coupelles planes pour différentes épaisseurs de couche.

On constate qu'au-dessous d'une certaine épaisseur, la couche reste affranchie de toute formation de craquelures. Cette épaisseur critique est propre au système constitué par la couche adhérant au support. Pour des couches d'épaisseur supérieure à la valeur critique, deux cas sont à distinguer : les couches plus minces dans lesquelles seuls des germes isolés sont présents (fig. 5a), et des couches plus épaisses où les craquelures envahissent toute la couche, formant un réseau dense et plus ou moins connecté (fig. $5 b$ et 5 c). Ces différentes figures de craquelures résultent de la compétition entre le processus de nucléation et celui de propagation d'une craquelure. En effet, la nucléation a lieu d'autant plus facilement que la couche est plus mince, donc plus sensible aux hétérogénéités. Dans le cas d'une couche mince, la propagation des craquelures est entravée par les hétérogénéités. Il en résulte que les couches comprenant de nombreux germes (fig. 5a) correspondent à des épaisseurs de l'ordre des tailles des hétérogénéités, alors que les couches présentant de longues craquelures, en plus faible nombre, correspondent à des couches épaisses (fig. 5c) [6]. La figure 5b montre une figure de craquelures relative à une épaisseur intermédiaire : un réseau de craquelures lisses présentant une dernière génération de craquelures sinueuses non connectées entre elles. 


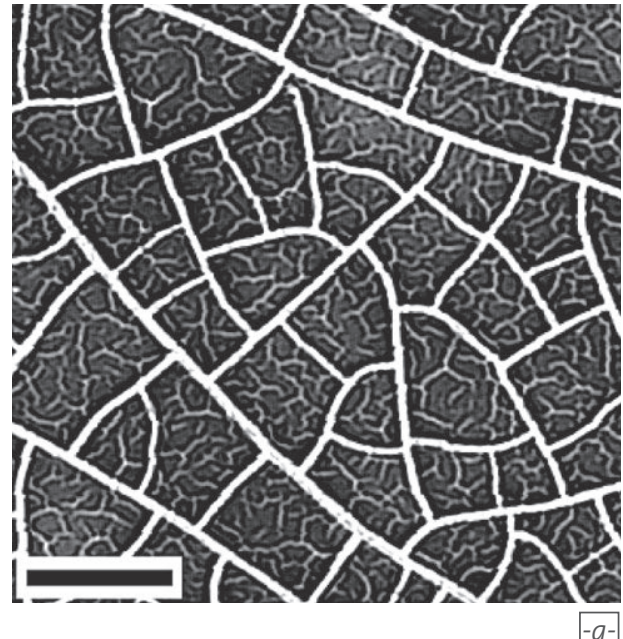

\section{Discussion}

Des expériences modèles, nous pouvons conclure que les réseaux de craquelures lisses, connectées entre elles (fig. 1a), sont caractéristiques d'une couche rigide plutôt épaisse, alors que les craquelures sinueuses, connectées entre elles et formant un réseau dense ou peu dense (fig. 1c), sont caractéristiques de la présence d'hétérogénéités. Une répartition de craquelures isolées comme le montre la figure 1d est, quant à elle, typique d'une couche ductile. En classant les différentes peintures de la série des Apôtres selon plusieurs catégories reliées à des figures de craquelures caractéristiques, nous obtenons le tableau 1. On constate que les peintures représentant Saint Jacques le Mineur et Saint Jude Thaddée ont pu être associées, tandis que la peinture de Saint Matthias ne peut être rapprochée d'une autre peinture de la série en question. Ceci recoupe la

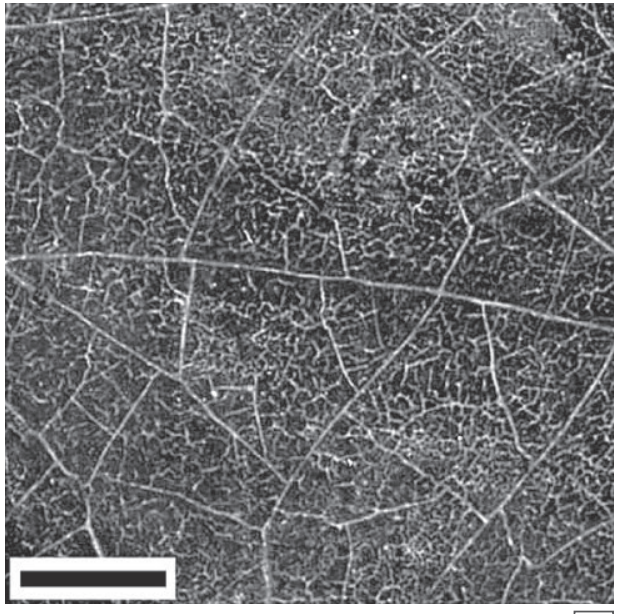

$-b-$

Figure 6 : Exemple de comparaison entre les expériences modèles et une morphologie de craquelures dans une couche picturale. L'image (a) a été réalisée en superposant les images des figures $5 a$ et $5 c$ : de longues craquelures lisses forment un réseau de polygones plus ou moins réguliers, dans lesquels de nombreux germes isolés sont observa bles. La morphologie obtenue peut être comparée, de manière qualitative, avec un extrait du réseau de craquelures dans la peinture de Saint Jacques le Mineur (b). Cette morphologie est analogue à ce que l'on pourrait observer dans le cas d'une couche mince craquelée, adhérant à une sous-couche. Les germes isolés sont uniquement présents dans la couche mince, tandis que le réseau de longues craquelures s'étend en profondeur de la couche superficielle à la couche sous-jacente.

(Longueur de la barre dans l'image (a) : 20 $\mathrm{m}$; longueur de la barre dans l'image (b) $: 1 \mathrm{~cm}$ ).

\section{Références}

Les informations que l'on peut obtenir en l'état actuel des connaissances sont encore qualitatives et partielles, notamment à cause de la complexité d'une œuvre picturale. En particulier, la diversité des morphologies de craquelures tient non seulement dans la délimitation de domaines polygonaux dans le plan de la couche mais aussi dans l'organisation des craquelures en profondeur, c'est-à-dire en tenant compte des sous-couches superposées. Néanmoins, la figure 6 montre qu'en superposant les réseaux de craquelures obtenus avec des monocouches, on peut retrouver de manière saisissante les réseaux de craquelures résultant de la superposition de couches picturales. Cette figure est à cet égard prometteuse des résultats que l'on peut attendre de telles études.

\begin{tabular}{|l|c|c|c|c|c|c|}
\hline & $\begin{array}{c}\text { craquelures } \\
\text { lisses }\end{array}$ & $\begin{array}{c}\text { craquelures } \\
\text { sinueuses }\end{array}$ & $\begin{array}{c}\text { réseau } \\
\text { peu dense }\end{array}$ & $\begin{array}{c}\text { réseau } \\
\text { dense }\end{array}$ & $\begin{array}{c}\text { craquelures } \\
\text { sinueuses non } \\
\text { connectées }\end{array}$ & $\begin{array}{c}\text { germes } \\
\text { isolés }\end{array}$ \\
\hline St Jude Thaddée & $\bullet$ & & & $\bullet$ & & \\
\hline St Jacques le Mineur & $\bullet$ & & & $\bullet$ & & \\
\hline St Jacques le Majeur & & & $\bullet$ & & $\bullet$ & \\
\hline St Paul & & & & $\bullet$ & & \\
\hline St Simon & & & & $\bullet$ & & \\
\hline St Matthias & & & & & & $\bullet$ \\
\hline St André & & & $\bullet$ & & & \\
\hline St Philippe & $\bullet$ & $\bullet$ & & $\bullet$ & & \\
\hline St Thomas & & $\bullet$ & $\bullet$ & & & \\
\hline St Pierre & $\bullet$ & $\bullet$ & & $\bullet$ & & \\
\hline
\end{tabular}

Tableau 1: Premiers résultats sur la classification des peintures des Apôtres selon différentes catégories de morphologies de craquelures. 